\title{
Volume entrained in the wake of a disk intruding into an oil-water interface
}

\author{
Ivo R. Peters,,${ }^{1,2,}$ Matteo Madonia, ${ }^{1}$ Detlef Lohse,,${ }^{1,3}$ and Devaraj van der Meer ${ }^{1}$ \\ ${ }^{1}$ Physics of Fluids Group, Faculty of Science and Technology, J.M. Burgers Center for Fluid Dynamics, \\ and MESA+ Institute, University of Twente, 7500 AE Enschede, Netherlands \\ ${ }^{2}$ Engineering and the Environment, University of Southampton, Highfield, \\ Southampton SO17 1BJ, United Kingdom \\ ${ }^{3}$ Max Planck Institute for Dynamics and Self-Organization, 37077 Göttingen, Germany
}

(Received 12 January 2016; published 1 July 2016)

\begin{abstract}
An object moving through a plane interface into a fluid deforms the interface in such a way that fluid from one side of the interface is entrained into the other side, a phenomenon known as Darwin's drift. We investigate this phenomenon experimentally using a disk which is started exactly at the interface of two immiscible fluids, namely, oil and water. First, we observe that due to the density difference between the two fluids the deformation of the interface is influenced by gravity and show that there exists a time window of universal behavior. Second, we show by comparing with boundary integral simulations that, even though the deformation is universal, our results cannot be fully explained by potential flow solutions. We attribute this difference to the starting vortex, which is created in the wake of the disk. Besides contributing significantly to entrainment directly, the vortex also influences the interface deformation due to Darwin's drift. Universal behavior is preserved, however, because the size and strength of the vortex shows the same universality as the potential flow solution.
\end{abstract}

DOI: 10.1103/PhysRevFluids.1.033901

\section{INTRODUCTION}

The flow around objects in a bulk medium is a classical problem in fluid dynamics which occurs in numerous natural phenomena and has many practical applications. The majority of research concerns high Reynolds number flows, where vortex shedding plays an important role, and mixing is greatly enhanced [1]. Until recently, and even though it was pointed out more than 60 years ago, much less attention has been given to mixing due to the drift volume, a mechanism described by potential flow known as Darwin's drift [2]. One of the recent studies has shown that for many marine animals Darwin's drift is a significant contributor to mixing by swimming [3]. Darwin's original proposition states that the drift volume induced by an object, which was started infinitely far away from an imaginary plane and moves infinitely far to the other side of the plane, is equal to the added mass volume of that object. His prediction is based on potential flow and was demonstrated explicitly for a circular cylinder [2]. It was confirmed and further generalized for a sphere two decades ago by Eames et al. [4], who added specific information concerning the method of evaluating the integrals to calculate the drift volume and of taking into account partial drift for finite systems. An open question is how the drift volume is influenced in the case of a less smooth object, or at higher Reynolds numbers, when flow separation starts to play a role. This problem is related to the drift volume of a vortex ring, which was considered analytically for a continuously expanding vortex [5] and experimentally for a steady vortex ring [6].

We approach this problem experimentally by impulsively starting a disk from the interface of two immiscible liquids, oil and water, similarly and with the same setup as we did before when studying the dynamics of an air-water interface when a disk would impact it [7-11]. Besides being a practical solution to studying drift volumes, this method has relevance for, e.g., the mechanism of oil

*i.r.peters@soton.ac.uk 


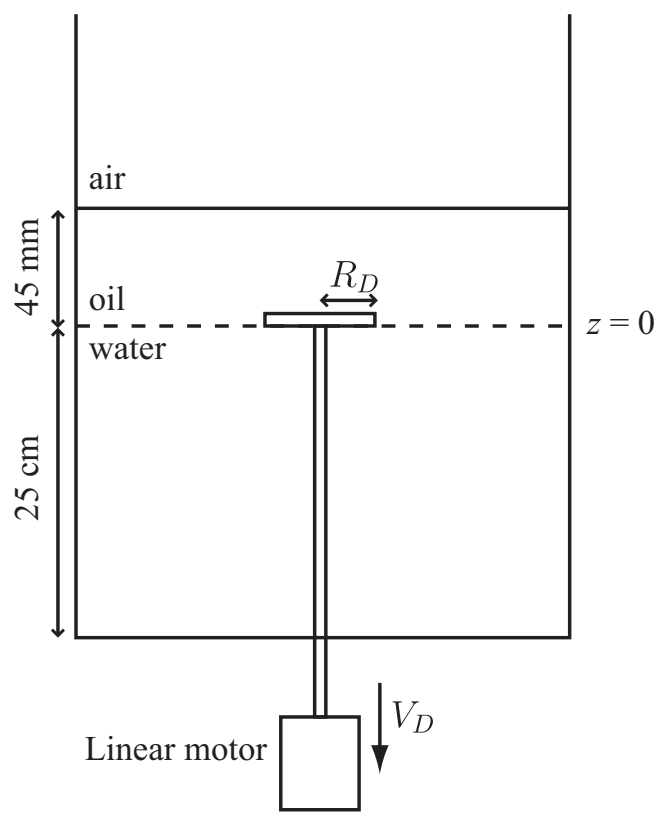

FIG. 1. Schematic view of the experiment, with disk radius $R_{D}$ and disk velocity $V_{D}$. We start with the bottom of the disk at rest at the interface between a deep layer of oil $(45 \mathrm{~mm})$ on top of a deep layer of water $(25 \mathrm{~cm})$, after which we pull down the disk at constant speed $V_{D}$. We define $z=0$ at the undisturbed oil-water interface.

dispersion in water [12]. In our experiment, when the disk moves down, it drags along the oil, which then obtains a particular funnel-shaped profile (see, e.g., Fig. 2). Both gravity and surface tension deform the shapes, and we determine how these profiles depend on the velocity of the disk. We observe that there exists a universal profile which becomes more prominent for higher velocities, for which this universal shape will extend deeper into the fluid. However, despite the observed universal behavior, these profiles do not agree with potential flow simulations we performed using a boundary integral technique. We attribute this difference to the formation of a vortex ring, which distorts the potential flow. We specifically show that the drift volume is larger than that predicted by potential flow.

\section{EXPERIMENTAL SETUP}

The experimental setup (Fig. 1) consisted of a water reservoir with a cross section of $15 \mathrm{~cm}$ by $15 \mathrm{~cm}$ and a height of $50 \mathrm{~cm}$. A linear motor that was mounted below the tank pulls a disk with a radius $R_{D}=20 \mathrm{~mm}$ through the water surface at a constant speed $V_{D}$, by means of a thin rod (radius $3 \mathrm{~mm}$ ) connecting the linear motor with the disk. The disk was accelerated with a maximum acceleration of $42 \mathrm{~m} / \mathrm{s}^{2}$ until the desired velocity $\left(V_{D}\right)$ was reached. The events were recorded with a Photron SA2 high-speed color camera at frame rates ranging from 1 to $8 \mathrm{kHz}$. The main control parameter of the experiment is an effective Froude number $\mathrm{Fr}^{*}$, which is similar to the regular Froude number defined as the disk speed $V_{D}$, made dimensionless using the disk radius $R_{D}$ and the gravitational acceleration $g$. Only we replace $g$ by the effective gravitational acceleration $g^{*}$ of the oil phase inside the water phase, as one would use to determine the wave speed of gravitational waves on a density interface $g^{*}=g\left(\rho_{w}-\rho_{o}\right) /\left(\rho_{w}+\rho_{o}\right)$ [13], yielding

$$
\mathrm{Fr}^{*}=\frac{V_{D}^{2}}{g R_{D}}\left(\frac{\rho_{w}+\rho_{o}}{\rho_{w}-\rho_{o}}\right),
$$




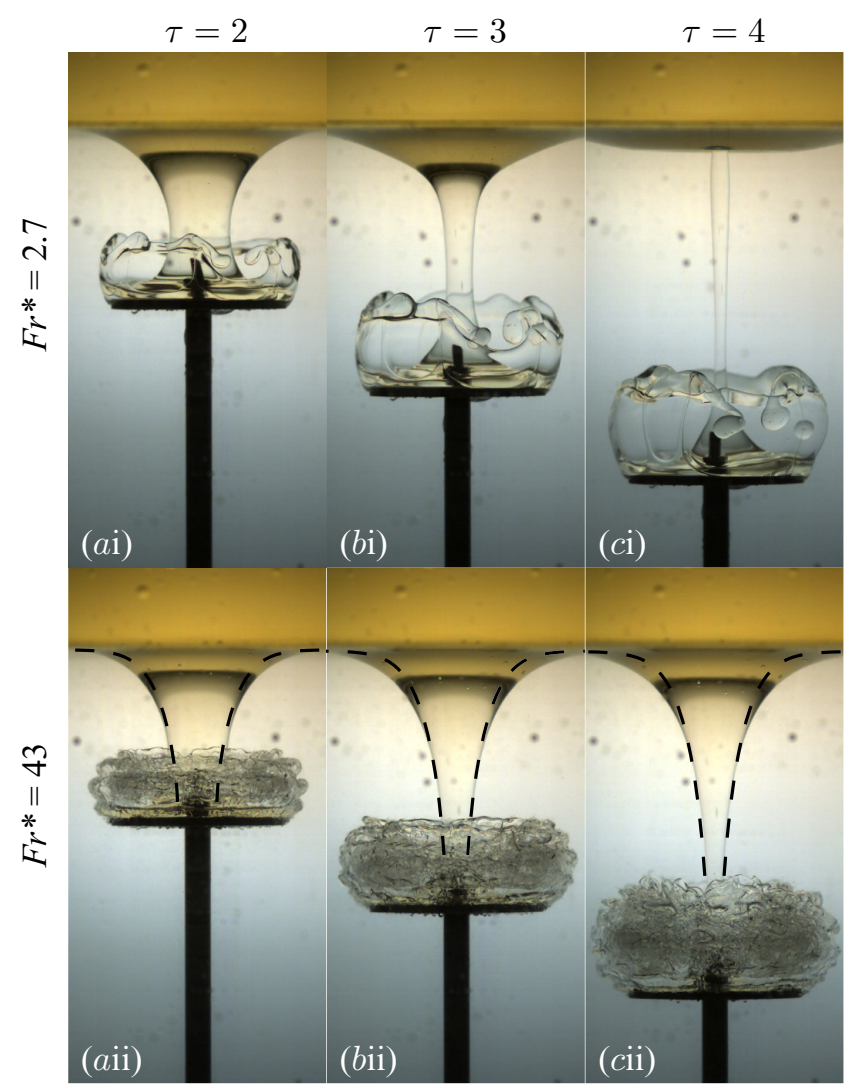

FIG. 2. Snapshots from experiments with two different values of the effective Froude number, (ai)-(ci) $\mathrm{Fr}^{*}=2.7$ and (aii) - (cii) $\mathrm{Fr}^{*}=43$. Corresponding pictures have been taken at the same dimensionless times $\tau=2, \tau=3$, and $\tau=4$. In the top experiment gravity has a clear influence on the shape of the entrained oil column. The bottom experiment is in the inertial regime, where gravity has negligible influence. A vortex ring is formed above the disk, which grows with the same dimensionless rate, independent of the Froude number. The black dashed line is the result of boundary integral simulations.

where $\rho_{w}$ and $\rho_{o}$ are the densities of water and oil, respectively. In our experiments we used sunflower oil, which has a density $\rho_{o}=900 \mathrm{~kg} / \mathrm{m}^{3}$ and a viscosity $v \sim 50 \times 10^{-6} \mathrm{~m}^{2} / \mathrm{s}$. Next to demineralized water we used a solution of table salt in water to increase the density of the water phase. We dissolved $1.0 \mathrm{~kg}$ of table salt in $5000 \mathrm{ml}$ water, resulting in $\rho_{s w}=1140 \mathrm{~kg} / \mathrm{m}^{3}$. The thickness of the oil layer in these experiments was $45 \mathrm{~mm}$, which was thick enough to be considered infinite. We verified this by performing the same experiment with increased oil layer thicknesses of 90 and $135 \mathrm{~mm}$, which did not influence our results.

\section{RESULTS}

Figure 2 shows two experiments in which we pulled down the disk from the oil-water interface at different velocities $V_{D}=0.25$ and $1.0 \mathrm{~m} / \mathrm{s}$. Initially the disk was at rest and its lower surface was aligned with the oil-water interface. Then, the disk was set into motion and in a short period of time obtained a constant speed $V_{D}$ [14]. A vortex ring appeared just above the disk, along with a smooth profile in the center which connects to the thick oil layer at the top. We first focus on this smooth profile, which has similarities with the shapes seen in [15], although that study concentrated on the formation of the vortex ring. 
In order to compare the experiments for different disk speeds, we define a dimensionless time $\tau=z(t) / R_{D}$, where $z(t)$ is the depth the disk has reached at time $t$ after starting from $z=0$ at $t=0$; i.e., at equal dimensionless times the disk has reached the same vertical position below the undisturbed oil-water interface, measured in units of the disk radius $R_{D}$. By varying the acceleration we verified that at equal target velocities $V_{D}$ and dimensionless times $\tau$ the observed column shapes are independent of the precise value of the acceleration used in experiments [16]. If the disk would be moving at a constant velocity $V_{D}$ all the time, i.e., if the acceleration phase would be infinitely small, then $\tau$ and $t$ would be related as $\tau=t V_{D} / R_{D}$. In the remainder of the article for simplicity we ignore the existence of the acceleration phase and take $z(t)=V_{D} t$.

Comparing Figs. 2(ai) and 2(aii), we see that at $\tau=2$ the shape of the entrained oil is very similar for $\mathrm{Fr}^{*}=2.7$ and $\mathrm{Fr}^{*}=43$, although the amount of vorticity in the vortex ring, as expected from dimensional analysis, appears to be much larger for the higher speed. At $\tau=3$ the effect of buoyancy becomes visible for the lower Froude number, where a difference in the shape of the entrained oil between Figs. 2(bi) and 2(bii) is appreciable. In the last frame, Figs. 2(ci) and 2(cii) at $\tau=4$, the oil in the case of $\mathrm{Fr}^{*}=2.7$ has clearly moved back up due to buoyancy, leaving only a relatively straight cylinder of oil behind. For $\mathrm{Fr}^{*}=43$, the shape is still unaffected by gravity at this point in time.

In order to see what the effect of the density difference is on the shape that we obtain at high Froude numbers, we performed experiments with demineralized water $\left(\rho=998 \mathrm{~kg} / \mathrm{m}^{3}\right)$ at $V_{D}=1 \mathrm{~m} / \mathrm{s}$ $\left(\mathrm{Fr}^{*}=99\right)$ and with salt water $\left(\rho=1140 \mathrm{~kg} / \mathrm{m}^{3}\right)$ at $V_{D}=1.5 \mathrm{~m} / \mathrm{s}\left(\mathrm{Fr}^{*}=97\right)$. Although there is a factor of 2 in the density difference between the oil and the water phases (demineralized vs salt water), the difference in the profiles is negligible, which leads us to conclude that the shape of the entrained oil column does not strongly depend on the relative density difference between the fluids. The use of salt water does, however, have an experimental advantage: The oil-water interface became less contaminated with oil and water droplets after the experiment was finished, which reduced the time that we had to wait between two experiments until the surface was smooth enough to clearly observe formation of the profile of the entrained oil. For experimental convenience, we used salt water in all experiments, except noted otherwise.

In Fig. 3 we compare the profiles of the entrained oil at equal dimensionless times $\tau$ and for a wide range of Froude numbers. Every experiment in Figs. 3(a)-3(c) consists of several repetitions of the experiment, sometimes using two different disk accelerations (21 and $\left.42 \mathrm{~m} / \mathrm{s}^{2}\right)$, indicating the excellent reproducibility of the experiment and the irrelevance of the initial startup motion of the disk and the actual acceleration that is used in this startup phase.

The appearance of differences in the shapes shown in Figs. 3(a)-3(c) is a result of gravity that is pushing the oil phase upwards. This will only happen if the time is long enough for gravity to become more important than the inertia that is pulling the oil phase down. We can predict the moment that differences appear by comparing the inertial time scale $t_{\text {in }} \equiv R_{D} / V_{D}$ to the gravitational time scale $t_{g} \equiv \sqrt{R_{D} / g^{*}}$. Gravity is expected to play a role only if $t \gtrsim t_{g}$, which, after dividing both sides by the inertial time scale, can be written as

$$
\tau^{2} \gtrsim \mathrm{Fr}^{*}
$$

where we have used $t / t_{\text {in }}=\tau$. If we now again look at Figs. 3(a)-3(c), we expect according to Eq. (2) to see a difference for $\mathrm{Fr}^{*} \lesssim 4$ at $\tau=2$, for $\mathrm{Fr}^{*} \lesssim 9$ at $\tau=3$, and for $\mathrm{Fr}^{*} \lesssim 16$ at $\tau=4$. These predictions agree well with the moment that we observe differences in the experimental profiles in Figs. 3(a)-3(c).

We now proceed to compare our experimental findings to potential flow solutions. For this, we use the boundary integral method as described in [8,11,17-19]. We performed boundary integral simulations of an impulsively started disk in an infinite bath of homogeneous fluid, moving at a constant speed $V_{D}$. The disk had the same size and thickness as in the experiment. We injected tracers at the position corresponding to the initial oil-water interface, i.e., aligned with the bottom of the disk. The tracers were then advected with the flow field around the disk. Because we used tracers in an infinite bath of a single liquid, the motion of the tracers corresponds to the case of $\mathrm{Fr}^{*} \rightarrow \infty$. 

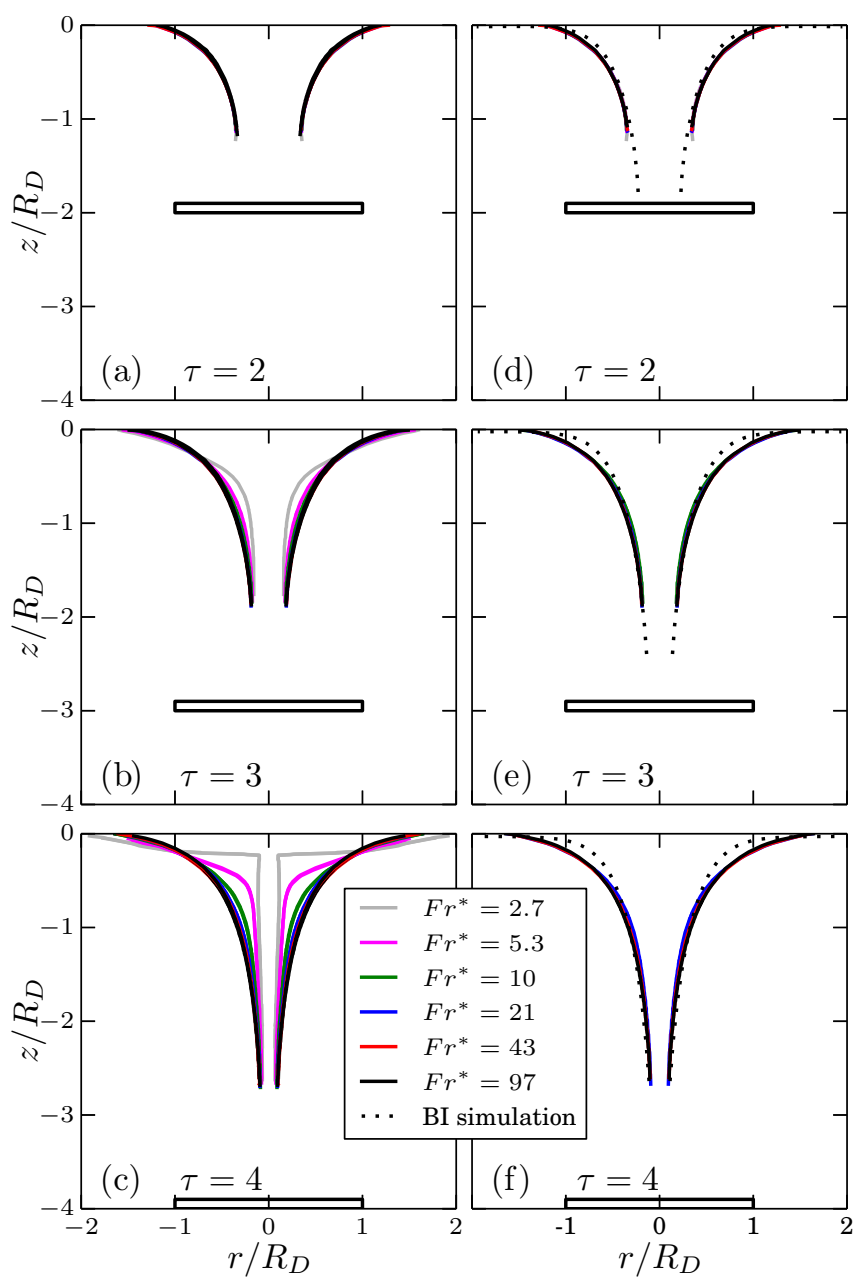

FIG. 3. Profiles of the entrained oil, for effective Froude numbers ranging from 2.7 to 97 . We observe universal profiles for $\mathrm{Fr}^{*} \rightarrow \infty$; each color in the image corresponds to one Froude number. (a) At $\tau=2$, all shapes collapse. (b) At $\tau=3$, a difference becomes visible for $\mathrm{Fr}^{*}=2.7$ and $\mathrm{Fr}^{*}=5.3$. (c) At $\tau=4$, for increasing $\mathrm{Fr}^{*}$, the shapes of the entrained oil converge to a single universal profile independent of $\mathrm{Fr}^{*}$. (d)-(f) The same profiles (only retaining shapes fulfilling the universality criterium $\tau^{2}<\mathrm{Fr}^{*}$ ) together with the boundary integral simulation results (dotted lines). The position and size of the disk is indicated by the black rectangle. The profile of the oil-water interface is not shown in the vicinity of the disk because the profile was not visible due to the vortex ring; see Fig. 2.

To validate the numerical code, we verified our simulations by calculating the displaced volume in the case of a sphere. We found good agreement with the analytical results of Eames et al. [4] for $\rho_{\max } /\left|x_{0}\right| \rightarrow \infty$, where $\rho_{\max }$ is the radius of the reference plane that is taken into account in the calculation of the drift volume and $x_{0}$ the initial axial distance of the sphere from this plane (see [4] for details). We note that in our experiments the initial position of the disk is at the reference plane (the oil-water interface), such that $x_{0} \rightarrow 0$, and, consequently, $\rho_{\max } /\left|x_{0}\right| \rightarrow \infty$.

Figures 3(d)-3(f) show that the shapes are approximated by our simulation results, but also that there exists a significant difference close to the undisturbed surface. A closer inspection reveals that the discrepancy increases as the disk moves farther down: while at $\tau=2$ [Fig. 3(d)] there is a 


\section{PETERS, MADONIA, LOHSE, AND VAN DER MEER}

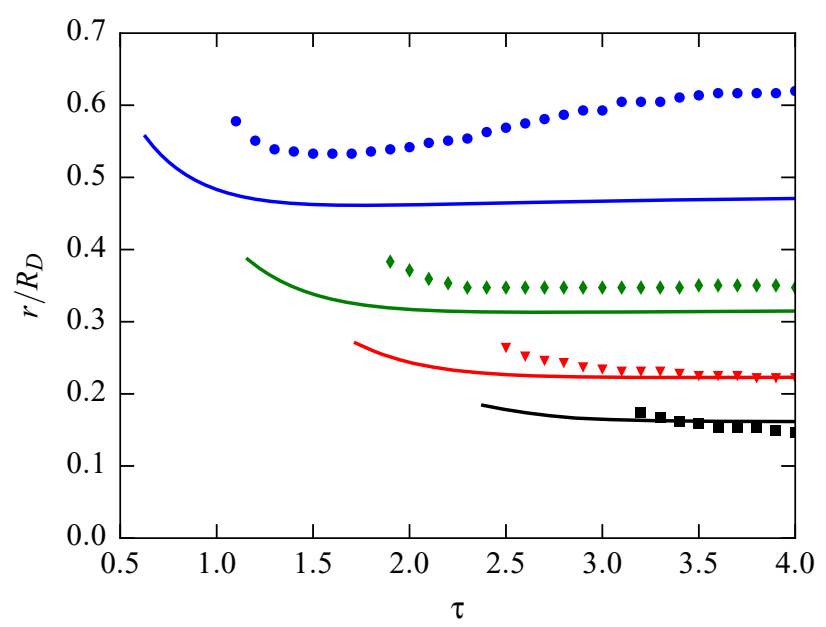

FIG. 4. Radius of the entrained oil column as a function of dimensionless time $\tau$ at different depths $z$ for the experiment (solid symbols) and the simulation (solid lines). Blue circles, $z / R_{D}=-0.5$; green diamonds, $z / R_{D}=-1.0$; red triangles, $z / R_{D}=-1.5$; black squares, $z / R_{D}=-2.0$.

reasonable agreement between the simulation and experiment, at $\tau=4$ [Fig. 3(f)] the difference is much larger. The same discrepancy is also illustrated in Figs. 2(aii)-2(cii).

We further investigate the development of this difference between the experiments and simulation in Fig. 4, where we plot the radius of the oil-water interface at different depths $z$ below the location of the undisturbed interface at $z=0$. First, we observe that the larger the distance to the undisturbed surface is, the smaller the discrepancy becomes. The difference in radius even appears to switch sign at the deepest position plotted here (black squares), although this difference is close to the experimental uncertainty. Second, the figure shows an apparent qualitative difference between the experiments and simulations at $z / R_{D}=-0.5$, for which the simulation shows an initial decrease of the radius, which then seemingly asymptotes to a constant value. The experiment, on the other hand, shows a significant increase in radius after the initial decrease [20].

A qualitative explanation for both these observations originates from the formation and growth of the vortex which introduces an additional downward velocity (and velocity gradient) inside the entrained volume of oil, since oil will continuously flow into the vortex. Where the shape of the entrained oil volume $r(z, t)$ is columnlike (i.e., $\partial r / \partial z$ is small), this velocity gradient will lead to simple stretching and thus a decreasing radius of the column [21]. However, where there exists a large gradient in the radius, the overall downward translation may introduce an increase in radius in the laboratory frame. This can be seen as follows. Because the volume $\propto r^{2} v_{z}$ of the entrained oil is conserved, the interface $r(z, t)$ obeys the partial differential equation

$$
\frac{\partial r^{2}}{\partial t}+\frac{\partial}{\partial z}\left(r^{2} v_{z}\right)=0
$$

where $v_{z}(z, t)(\geqslant 0)$ is the downward velocity in the vertical direction inside the column, which for simplicity is assumed to be independent of the radial coordinate. This can immediately be rewritten as

$$
\frac{\partial r}{\partial t}+v_{z} \frac{\partial r}{\partial z}+\frac{1}{2} r \frac{\partial v_{z}}{\partial z}=0 .
$$

Note that for such a stretching flow the second term in the above expression is always negative, since $\partial r / \partial z<0$ for the measured profiles, whereas the third (or stretching) term is always positive because $\partial v_{z} / \partial z \geqslant 0$ in the entrained columnlike shape and under the universality condition $\tau^{2}<\mathrm{Fr}^{*}$. In the 
case of a purely columnar shape, the second term is zero, and therefore $\partial r / \partial t$ is negative. In the case of a funnel-shaped profile with a strong radial gradient $(\partial r / \partial z \ll 0)$ and sufficient downward velocity, the magnitude of the second term may well be larger than the stretching term, which will result in a positive $\partial r / \partial t$.

The remaining question is why we still observe universal shapes even though there is a clear influence from the vortex. A possible explanation for this can be found in [22], where it is shown that for early times $(\tau \lesssim 8)$ the dimensionless size and strength of a vortex behind an impulsively started disk in a homogeneous liquid is independent of the disk speed. Although in our case the fluid is not homogeneous and the velocities are higher, visual inspection suggests that the same holds for our experiments: In Fig. 2, the vortex for $\mathrm{Fr}^{*}=2.7$ has the same size as that for $\mathrm{Fr}^{*}=43$, when they are compared at the same dimensionless time.

We now proceed to providing an estimate of the magnitude of the downward velocity $v_{z}$ due to the presence of the vortex. Given the circulation $\Gamma$ of a vortex line in a closed loop $C$, the velocity $\mathbf{v}$ at position $\mathbf{r}$ can be obtained from the Biot-Savart law

$$
\mathbf{v}(\mathbf{r})=\frac{\Gamma}{4 \pi} \oint_{C} \frac{d \mathbf{l} \times(\mathbf{l}-\mathbf{r})}{|\mathbf{l}-\mathbf{r}|^{3}}
$$

where $\mathbf{l}$ is the core of the vortex line. For a ring-shaped vortex with a radius equal to the disk radius $R_{D}$ we can write for points $\mathbf{r}$ on the axis of symmetry that, taking into account that the velocity is purely vertical, $|\mathbf{l}-\mathbf{r}|=\sqrt{R_{D}^{2}+\left(z^{\prime}\right)^{2}}$ and $\hat{\mathbf{e}}_{\mathbf{z}} \cdot(d \mathbf{l} \times(\mathbf{l}-\mathbf{r}))=R_{D} d l$, with $\hat{\mathbf{e}}_{\mathbf{z}}$ the unit vector in the $z$ direction, $d l=R_{D} d \theta$, and $z^{\prime}=z-z_{c}$, where $z_{c}$ is the vertical position of the core of the vortex. The $z$ component of the integral of Eq. (5) can now be evaluated straightforwardly as

$$
v_{z}\left(z^{\prime}\right)=\frac{\Gamma}{4 \pi} \int_{0}^{2 \pi} \frac{R_{D}^{2}}{\left(R_{D}^{2}+z^{\prime 2}\right)^{3 / 2}} d \theta
$$

and gives the vertical component of the velocity $v_{z}$ as

$$
v_{z}=\frac{R_{D}^{2} \Gamma}{2\left(R_{D}^{2}+z^{\prime 2}\right)^{3 / 2}},
$$

or, in dimensionless form,

$$
\tilde{v}_{z}=\frac{\tilde{\Gamma}}{2\left(1+\tilde{z}^{\prime 2}\right)^{3 / 2}},
$$

where $\tilde{v}_{z}=v_{z} / V_{D}, \tilde{z}=z / R_{D}$, and the dimensionless, time-dependent circulation $\tilde{\Gamma}=\Gamma /\left(R_{D} V_{D}\right)$ is independent of the disk speed [22]. In Fig. 5 we compare the vertical velocity on the axis of symmetry resulting from our potential flow calculation to the one induced by the vortex ring at different instances of time. We have used the empirical relation from Yang et al. [22] for $\tilde{\Gamma}(\tau)$ [23] and approximated the velocity of the vortex core to be linear (i.e., $\tilde{z}^{\prime}=\tilde{z}_{D}+0.25 \tau$ ). The latter approximately matches the position of the vortex core in the experiments, where we observe that the core is about one disk radius above the disk at $\tau=4$ (see Fig. 2). Clearly, the velocities and velocity gradients are greatly enhanced by the starting vortex, and an influence on the shape of the entrained oil column is therefore expected. Most importantly we note that the velocity profiles of Fig. 5 are independent of the disk velocity, resulting in a mechanism through which the universality of the shapes shown in Fig. 3 are preserved.

To further quantify the influence of the vortex on the drift volume, we calculate the entrained volume of oil in both the simulations and the experiment. Because a part of the interface is masked by the vortex, we do not calculate the complete displaced volume [4], but only compare the part that is accessible in both the experiment and the simulation. In the simulations we find, at $\tau=4$, between the depths $z / R_{D}=-0.2$ and $z / R_{D}=-1.0$ (with $z=0$ at the unperturbed oil-water interface), an entrained volume of $4.24 \mathrm{ml}$, while in the experiment $7.25 \mathrm{ml}$ of oil is entrained. Extending the 


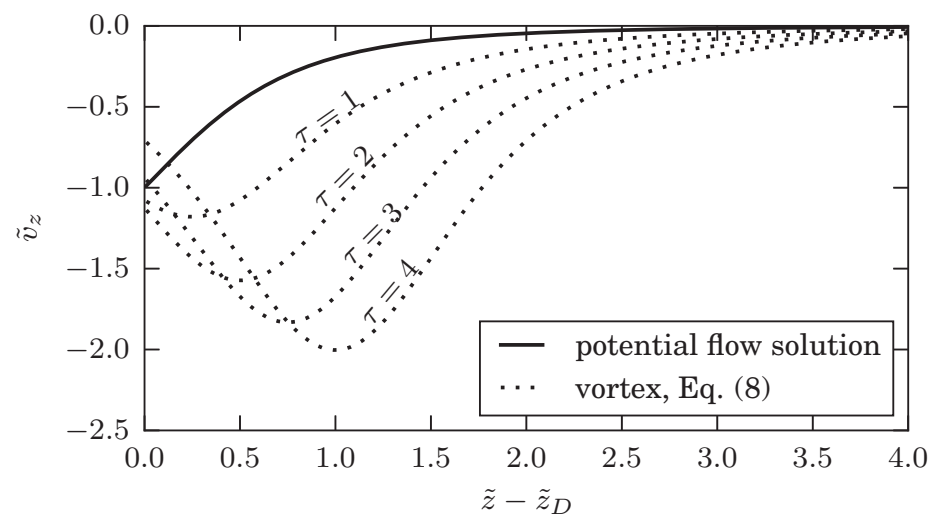

FIG. 5. Comparison of the estimated nondimensional velocity $\tilde{v}_{z}=v_{z} / V_{D}$ on the axis of symmetry as a function of the nondimensional distance $\tilde{z}-\tilde{z}_{D}=\left(z-z_{D}\right) / R_{D}$ to the center of the disk for the potential flow solution (solid line) and the time-dependent contribution from the vortex ring (dotted lines) at four different instances in dimensionless time $\tau=t R_{D} / V_{D}$.

range to $z / R_{D}=-2.0$ gives $5.57 \mathrm{ml}$ and $8.66 \mathrm{ml}$ for the simulation and experiment, respectively. Clearly, the experimental volume of entrained oil is significantly larger than the volume predicted by the potential flow simulations.

\section{CONCLUSIONS AND OUTLOOK}

We have performed experiments where we started a disk at an oil-water interface and pulled it down at a constant speed. We have shown that at high speeds, gravity and surface tension can be neglected, and the entrained oil obtains a universal funnel shape, independent of the Froude number. However, a vortex ring is formed at the disk edge, which influences the shape of the entrained oil, resulting in a qualitative and quantitative difference compared to the potential flow solution. Nonetheless, the universality of the funnel shape is conserved. The shape also appears insensitive to the relative density difference, at least for the density differences studied.

Our experiments may be compared to other studies that investigate the entrainment of one liquid into another at relatively small density differences. One example is the entrainment induced by bubbles rising through a liquid-liquid interface [24]. Although the situation is more complicated because of the deformation of the bubble and the speed is not a direct control parameter, universal interface shapes might exist under some conditions for large enough bubble velocities.

The effect might be investigated further for flows at lower Reynolds numbers, exploring the limit where the vortex disappears. In our current setup this regime is inaccessible due to the influence of gravity at low disk speeds. Scenarios with a reduced influence of the vortex can alternatively be explored by replacing the disk with an object without sharp edges (e.g., a sphere) such that flow separation is suppressed. A consequence of our finding is that the displaced volume as predicted by [2] is underestimated in cases where flow separation is of importance, in addition to the increased mixing as a direct consequence of the vortex. This introduces a nontrivial shape effect on the entrainment in the wake of, for example, ocean life [3]. The preserved universality in such cases, however, may help in simplifying and generalizing analysis in these situations.

\section{ACKNOWLEDGMENTS}

We acknowledge financial support from FOM and the NWO-Spinoza program. 
[1] H. Choi, W.-P. Jeon, and J. Kim, Control of flow over a bluff body, Annu. Rev. Fluid Mech. 40, 113 (2008).

[2] C. Darwin, Note on hydrodynamics, Math. Proc. Cambridge Philos. Soc. 49, 342 (1953).

[3] K. Katija and J. O. Dabiri, A viscosity-enhanced mechanism for biogenic ocean mixing, Nature (London) 460, 624 (2009).

[4] I. Eames, S. E. Belcher, and J. C. R. Hunt, Drift, partial drift and Darwin's proposition, J. Fluid Mech. 275, 201 (1994).

[5] J. S. Turner, The flow into an expanding spherical vortex, J. Fluid Mech. 18, 195 (1964).

[6] J. O. Dabiri, Note on the induced Lagrangian drift and added-mass of a vortex, J. Fluid Mech. 547, 105 (2006).

[7] R. Bergmann, D. van der Meer, M. Stijnman, M. Sandtke, A. Prosperetti, and D. Lohse, Giant Bubble Pinch-Off, Phys. Rev. Lett. 96, 154505 (2006).

[8] R. Bergmann, D. van der Meer, S. Gekle, A. van der Bos, and D. Lohse, Controlled impact of a disk on a water surface: Cavity dynamics, J. Fluid Mech. 633, 381 (2009).

[9] S. Gekle, J. M. Gordillo, D. van der Meer, and D. Lohse, High-Speed Jet Formation after Solid Object Impact, Phys. Rev. Lett. 102, 034502 (2009).

[10] S. Gekle, I. R. Peters, J. M. Gordillo, D. van der Meer, and D. Lohse, Supersonic Air Flow due to Solid-Liquid Impact, Phys. Rev. Lett. 104, 024501 (2010).

[11] S. Gekle and J. M. Gordillo, Generation and breakup of Worthington jets after cavity collapse. Part 1. Jet formation, J. Fluid Mech. 663, 293 (2010).

[12] D. W. Murphy, C. Li, V. D'Albignac, D. Morra, and J. Katz, Splash behaviour and oily marine aerosol production by raindrops impacting oil slicks, J. Fluid Mech. 780, 536 (2015).

[13] P. K. Kundu and I. M. Cohen, Fluid Mechanics, 3rd ed. (Elsevier, Amsterdam, 2004).

[14] With an acceleration of $42 \mathrm{~m} / \mathrm{s}^{2}$, it takes $0.024 \mathrm{~s}$ to reach $V_{D}=1.0 \mathrm{~m} / \mathrm{s}\left(\mathrm{Fr}^{*}=43\right)$. The duration of the experiment in that case is $0.08 \mathrm{~s}$. The acceleration does not significantly influence the experiment, as can be appreciated in Fig. 3.

[15] Q.-X. Lian and Z. Huang, Starting flow and structures of the starting vortex behind bluff bodies with sharp edges, Exp. Fluids 8, 95 (1989).

[16] We have used accelerations ranging from 21 to $42 \mathrm{~m} / \mathrm{s}^{2}$ and found no discernible differences in the shape of the entrained oil column, up to the point in time that gravity becomes significant, where small differences would be introduced due to the somewhat longer time that is needed to reach a depth $z(t)$ at small accelerations.

[17] H. N. Oguz and A. Prosperetti, Dynamics of bubble growth and detachment from a needle, J. Fluid Mech. 257, 111 (1993).

[18] S. Gekle and J. M. Gordillo, Compressible air flow through a collapsing liquid cavity, Int. J. Numer. Methods Fluids 67, 1456 (2011).

[19] C. Pozrikidis, Introduction to Theoretical and Computational Fluid Dynamics (Oxford University Press, New York, 2011).

[20] Actually, also in the simulations we observe such an increase, only much smaller $(<2 \%$ of the minimum radius) than in the experiment.

[21] J. Eggers and E. Villermaux, Physics of liquid jets, Rep. Prog. Phys. 71, 036601 (2008).

[22] A. Yang, L. Jia, and X. Yin, Formation process of the vortex ring generated by an impulsively started circular disc, J. Fluid Mech. 713, 61 (2012).

[23] $\Gamma \approx-4.67\left(1-0.75 e^{-0.416 \tau}-(1-0.75) e^{-24.927 \tau}\right)$.

[24] R. Bonhomme, J. Magnaudet, F. Duval, and B. Piar, Inertial dynamics of air bubbles crossing a horizontal fluid-fluid interface, J. Fluid Mech. 707, 405 (2012). 